ISSN: 2386-3919

ISSN electrónico: 2386-3927

DOI: http://dx.doi.org/10.14201/et2015332171190

\title{
PERCEPCIÓN DE LOS ESTUDIANTES DEL GRADO EN PEDAGOGÍA Y EDUCACIÓN SOCIAL SOBRE LA ORGANIZACIÓN, LA UTILIDAD Y FORMACIÓN DEL PRACTICUM
}

\section{Students perception about Pedagogy and Social Education degree related to the Practicum organization, usefulness and training}

\section{Aperçu des étudiants du Diplôme en Pédagogie et Éducation Sociale sur l'organisation et l'utilité de leur formation stagiaire}

Sonia CASILlas MarTíN*, Margarita GONZÁlEz SÁNCHEZ*** y Sara SERRATE GonZÁleZ****

* Departamento Didáctica, Organización y MIDE. Facultad de Educación.

Universidad de Salamanca. Correo-e: scasillasma@usal.es

* Departamento Teoría e Historia de la Educación. Facultad de Educación.

Universidad de Salamanca. Correo-e: mgsa@usal.es

*** Facultad de Educación. Universidad de Salamanca. Correo-e: sarasg@usal.es

Recibido: 23-03-2015; Aceptado: 20-05-2015; Publicado: 30-10-2015

BIBLID [2386-3927 (2015) 33, 2; 171-190]

Ref. Bibl. SONIA CASILLAS MARTÍN, MARGARITA GONZÁLEZ SÁNCHEZ y SARA SERRATE GONZÁlEZ. Percepción de los estudiantes del Grado en Pedagogía y Educación Social sobre la organización, la utilidad y formación del Practicum. Enseñanza \& Teaching, 33, 2-2015, 171-190. http://dx.doi.org/10.14201/ et2015332171190 
SONIA CASILLAS MARTÍN, MARGARITA GONZÁLEZ SÁNCHEZ Y SARA SERRATE GONZÁLEZ PERCEPCIÓN DE LOS ESTUDIANTES DEL GRADO EN PEDAGOGÍA Y EDUCACIÓN SOCIAL SOBRE LA ORGANIZACIÓN, LA UTILIDAD Y FORMACIÓN DEL PRACTICUM

RESUMEN: El presente artículo muestra los principales resultados de un estudio que persigue conocer el grado de satisfacción con el proceso seguido para asignar centro o institución de prácticas, la percepción previa respecto a la utilidad de las prácticas externas de titulación y de la formación para el Practicum de los estudiantes de Pedagogía y Educación Social de la Facultad de Educación de la Universidad de Salamanca. Para la realización del estudio de tipo descriptivo-correlacional, se ha utilizado un cuestionario, creado ad hoc, aplicado a través de Internet. La muestra participante respondió al cuestionario una vez finalizado el periodo formativo de la asignatura Practicum y antes de comenzar las prácticas externas en las entidades colaboradoras. Los resultados nos han permitido identificar que el alumnado, en general, se muestra satisfecho con la asignación del centro en prácticas, siendo la satisfacción más elevada en la titulación de Educación Social. Los estudiantes consideran que las prácticas externas les van a permitir aplicar actitudes hacia la profesión, aprender y aplicar habilidades interpersonales, desarrollar competencias y capacidades importantes y conocer mejor el ámbito profesional de su titulación. Los estudiantes de ambas titulaciones creen que la formación en la asignatura Practicum y a lo largo de ambos Grados es insuficiente y no les prepara para hacer frente a las prácticas externas. Entre las conclusiones más relevantes destacar que el alumnado considera que la gestión y organización de las prácticas es un proceso bien organizado, y con ello se determina que se encuentran motivados y con una predisposición positiva para realizar las prácticas. No obstante, hay que tener en cuenta que es necesario introducir determinadas mejoras en torno a los criterios de asignación del centro e iniciar planes de formación práctica más exhaustivos en relación a formación inicial de los futuros pedagogos y educadores sociales.

Palabras clave: prácticas; formación profesional; Pedagogía; Educación Social; percepción; satisfacción profesional.

SUMMARY: The current article shows the main results obtained from a study which aim was to know the student body's valuation with respect to the process of development and management of the subject called Practicum, the assessment criteria, the initial perception related to the external practices utility and the evaluation students do about the training acquired to confront the practices of the degree.

For the development of the study, which has a descriptive correlational design, a questionnaire created ad hoc and applied through the Internet was used. The participating sample filled out the questionnaire before starting the external practices. A descriptive and exploratory analysis was carried out throughout the SPSS v.21 statistical method for the interpretation of the data.

The results confirm that, in general, the students who participate in this study seem to be satisfied with the centre assigned to do the practices. Among the utility criteria that the external practices will yield, it is noticed that students believe that this period will let them to create and develop professional attitudes, to learn and employ the typical interpersonal abilities of a Pedagogue or Educator, to develop practical competences and aptitudes as well as they will offer them a wider vision of the work setting related to their degree. Regarding to the training received to confront the external practices, students of both qualifications express that it is inadequate in the 
SONIA CASILLAS MARTÍN, MARGARITA GONZÁLEZ SÁNCHEZ Y SARA SERRATE GONZÁLEZ PERCEPCIÓN DE LOS ESTUDIANTES DEL GRADO EN PEDAGOGÍA Y EDUCACIÓN SOCIAL SOBRE LA ORGANIZACIÓN, LA UTILIDAD Y FORMACIÓN DEL PRACTICUM

sense that they do not feel qualified to deal with this new situation that is presented: learning from the own personal reality.

Key words: practice; vocational training; Pedagogy; Social Education; perception; professional satisfaction.

RÉSUMÉ: Ce document présente les principaux résultats d'une étude qui cherche à comprendre l'évaluation des étudiants concernant le processus et la gestion du "cours stage", leurs critères d'évaluation, leurs aperçus initiales concernant l'utilité des stages et de l'évaluation qui réalisent sur la formation réçue, pour faire face aux practiques de l'obtention du diplôme.

Pour aboutir à cette étude, descriptive-correlationnelle, nous avons utilisé un questionnaire créé ad hoc et appliqué à travers l'internet. L'échantillon des participants au procesus à répondu au questionnaire avant de commencer leurs pratiques à l'extérieur. Une analyse descriptive et exploratoire par SPSS v.21 a été réalisée pour l'analyse des données.

Les résultats nous permettent d'affirmer que les étudiants participants à cette étude étaient généralement satisfaits du centre auquel ils avaient été affectés pour l'accomplissement de cette tâche.

Parmi les bénéfices qui leur apportent ces pratiques externes, nous avons vérifié que les étudiants sont de l'avis que cette période leur permettra d'ameliorer leur formation et de développer des attitudes professionnelles, ainsi que d'apprendre et d'appliquer leur propre pédagogie, et de même cette pratique leur offrira une large vision sur le domaine de leur proffesion.

Quant à la formation reçue pour faire face aux pratiques externes, les étudiants affirment que c'est insuffissant dans le sens que, eux mêmes, considérènt qu'ils ne se voient pas capables pour faire face à cette nouvelle situation qui se présente devant eux.

Mots clés: pratique; formation professionnelle; Pédagogie; Éducation Sociale; aperçu, satisfaction professionnelle.

\section{INTRODUCCIÓN}

La materia Practicum es una asignatura formativa que potencia los conocimientos, habilidades y actitudes teóricos del alumno, y que comporta la aplicación de propuestas educativas y socioeducativas en contextos profesionales específicos reales. Es por ello que las prácticas externas suponen una «vía para superar la posible fragmentación del conocimiento adquirido a lo largo de las diferentes disciplinas» (Bas, 2005: 192) a través de una estrecha relación y articulación entre la teoría y la práctica profesional, que permite al alumnado la posibilidad de "Construir un pensamiento práctico" (Morales, 2013: 350) a través del desarrollo de instrumentos, recursos y formación específica que permiten ir formando una 
SONIA CASILLAS MARTÍN, MARGARITA GONZÁLEZ SÁNCHEZ Y SARA SERRATE GONZÁLEZ PERCEPCIÓN DE LOS ESTUDIANTES DEL GRADO EN PEDAGOGÍA Y EDUCACIÓN SOCIAL SOBRE LA ORGANIZACIÓN, LA UTILIDAD Y FORMACIÓN DEL PRACTICUM

identidad profesional propia, necesaria para su posterior inserción en el mundo laboral (López e Hinojosa, 2008).

En las titulaciones de Pedagogía y Educación Social, el Practicum es un elemento esencial en su formación universitaria, "será en el encuentro entre la teoría y la práctica donde podrá construir su propio conocimiento, aprender a actuar, asumir actitudes profesionales» (González, 2009: 36), puesto que supone un acercamiento y complemento del aprendizaje académico, más teórico, a la realidad profesional de cada titulación (González y Hevia, 2011). Proporciona al alumnado un espacio y tiempo formativo desde el que poder aprender a partir de la observación, reflexión y acción en un contexto determinado, facilitando la comprensión de las peculiaridades de la intervención propias de cada titulación. No obstante, la importancia del Practicum no radica únicamente en las posibilidades formativas que ofrece al alumno en el desarrollo de sus competencias sino que, en tanto espacio profesionalizador, supone una oportunidad para definir y redefinir los ámbitos profesionales de cada titulación, pasando, en el caso de Pedagogía, de una visión más escolar, a la inclusión en espacios más polivalentes (Zabalza, 2006), y en el caso de la Educación Social, además de un enriquecimiento del saber como del saber hacer, propiciando la consolidación de nuevos ámbitos y espacios laborales desde los que se demanda este perfil profesional (Sáez, 2009).

Estudios recientes como los realizados por Zabalza (2011) y Armengol et al. (2011) ponen de manifiesto que es necesario realizar evaluaciones exhaustivas de la formación para el Practicum que ofrece la Universidad y la formación que proporcionan los centros e instituciones profesionales que acogen al alumnado, en un intento de mantener una interrelación positiva que ayude a configurar una asignatura más adaptada y específica a las demandas y necesidades educativas y sociales actuales. Sin dejar a un lado la necesidad de evaluar el proceso y el resultado formativo que suponen las prácticas para el alumno, nuestro estudio centra su atención en conocer y analizar la percepción y la predisposición que posee y manifiesta el alumnado hacia la formación práctica, antes del inicio del periodo de prácticas externas (González, 2007; Latorre, 2007; Woolfolk y Murphy, 2001) de la titulación de Pedagogía y Educación Social.

Entre los aspectos contemplados, uno de ellos se dirige a conocer la valoración que el alumno manifiesta respecto al proceso de gestión y organización del Practicum. Desde la Universidad, se cuenta con una estructura organizativa en torno a la coordinación del Practicum que, aunque dispone de una amplia autonomía institucional (Gairín y Martín, 2004), debe asumir, en un acto de responsabilidad, un conjunto de tareas en torno a la gestión de las prácticas externas. El abanico de funciones a desempeñar para el buen funcionamiento del Practicum abarca desde la gestión de la información al alumno, la canalización de la oferta de plazas, hasta la adjudicación de las mismas. En este sentido, es importante conocer la percepción del alumnado en torno a este proceso, en un intento de verificar si el trabajo de elección y asignación de centro en prácticas repercute en la motivación y predisposición del alumnado a realizar las prácticas en la institución externa 
SONIA CASILLAS MARTÍN, MARGARITA GONZÁLEZ SÁNCHEZ Y SARA SERRATE GONZÁLEZ SOBRE LA ORGANIZACIÓN, LA UTILIDAD Y FORMACIÓN DEL PRACTICUM

adjudicada. El contento o descontento manifestado por el alumnado durante este periodo puede proporcionarnos una información valiosa del trabajo desempeñado por la coordinación de prácticas y, en este sentido, introducir mecanismos y herramientas que mejoren, en su caso, el proceso.

Para la organización de las prácticas externas en la Universidad de Salamanca, se cuenta con una comisión de prácticas que abarca las distintas titulaciones, en nuestro caso, se hace cargo de las Prácticas del Grado en Pedagogía y Educación Social. El proceso se inicia teniendo en cuenta que el perfil profesional para cada una de las titulaciones es diferente, puesto que, en el Grado en Pedagogía, se contemplan dos menciones para la realización de las prácticas, como son la mención de Formación y Gestión de calidad y la mención de Orientación educativa y Asesoramiento, mientras que en Educación Social no se dan itinerarios formativos de especialización. Las prácticas se llevan a cabo durante el cuarto curso teniendo una carga de 24 créditos ECTS en Pedagogía y de 30 créditos ECTs en Educación Social.

La formación previa al periodo de prácticas en los centros se desarrolla a través de seminarios impartidos por los responsables de diversas instituciones profesionales colaboradoras con las prácticas universitarias. Con estos encuentros, se pretende que el alumnado conozca diversos ámbitos de intervención profesional relacionados con el Grado y descubra aspectos esenciales, principalmente teóricos, del trabajo que el pedagogo/educador realiza en cada ámbito profesional. También se ofrece a los estudiantes lecturas relacionadas con diversos aspectos como la ética y el código deontológico, las funciones del pedagogo y educador social, actitudes hacia la profesión, normativa específica, características de los colectivos de intervención, recursos existentes, etc., sobre las que reflexionan en un portafolios que posteriormente es material de evaluación (Morales, 2010).

Al alumnado de las titulaciones objeto de estudio, se le ofrece la posibilidad de buscar y gestionar plaza en aquellos centros en los que les gustaría realizar sus prácticas y con los que la Universidad no tiene reservadas plazas para el Practicum y a los que podrán incorporarse una vez aceptados y firmado el convenio de cooperación correspondiente. Posteriormente, desde la Universidad se realiza una estimación de oferta de plazas y del alumnado matriculado cada curso académico. Para aquellos estudiantes que no hayan gestionado su plaza previamente, se realiza una difusión de la información sobre las entidades colaboradoras de las prácticas con la Universidad y se presenta una oferta de plazas disponibles en aquellos centros que tienen convenio con la Facultad, ofreciéndoles un abanico amplio de centros donde pueden hacer sus prácticas externas. El procedimiento de adjudicación de plaza se realiza en base a criterios de transparencia, asignando los centros de acuerdo al orden de preferencia que indique el alumno. En los centros más demandados, se utiliza como criterio para asignar cada plaza la nota media del alumno en Pedagogía o bien sorteo público ante el alumnado que ha elegido ese puesto en el caso de Educación Social.

Con una oferta de plazas superior a los estudiantes potenciales del Practicum, entre la variedad de centros e instituciones ofertadas se encuentran: 
SONIA CASILLAS MARTÍN, MARGARITA GONZÁLEZ SÁNCHEZ Y SARA SERRATE GONZÁLEZ PERCEPCIÓN DE LOS ESTUDIANTES DEL GRADO EN PEDAGOGÍA Y EDUCACIÓN SOCIAL SOBRE LA ORGANIZACIÓN, LA UTILIDAD Y FORMACIÓN DEL PRACTICUM

a) Centros de formación y gestión: Inspección Educativa, Instituto de Ciencias de la Educación, Programas propios de la Universidad de Salamanca, Consejerías de Educación, Diputación y Ayuntamientos.

b) Departamentos de Orientación de Institutos de Enseñanza Secundaria y Centros de Infantil y Primaria.

c) Gabinetes y equipos psicopedagógicos en instituciones y entidades públicas y privadas.

d) Centros especializados en intervención social con personas con discapacidad, drogodependientes, inmigrantes, tercera edad, en protección y promoción de la mujer, desarrollo comunitario, en menores y jóvenes, prevención de la delincuencia, ocio y tiempo libre, centros hospitalarios, etc.

Entre las múltiples y diversas instituciones y asociaciones, tanto nacionales e internacionales como municipales, que colaboran con la Universidad se encuentran Cruz Roja, Cáritas, Proyecto Hombre, ASCOL, AFEM, Insolamis, AFIM, Aspar la Besana, entre otras, con programas de calle, apoyo familiar, inserción sociolaboral, terapia de grupo, desarrollo de habilidades sociales, reinserción de toxicómanos, apoyo educativo y extraescolar, etc.

Una vez que el alumno conoce el centro que le ha sido asignado, y previo al inicio del periodo de prácticas externas, se informa al alumnado de los criterios de evaluación seguidos en la asignatura Practicum. Se tienen en cuenta, por un lado, los documentos elaborados por los estudiantes, como son un portafolios y una memoria, documentos que pretenden fomentar la reflexión crítica del alumno desde una metodología centrada en el aprendizaje (Martínez, 2007), así como recoger las evidencias que antes, durante y después de las prácticas hayan supuesto momentos y experiencias de aprendizaje para el alumno. Estos documentos, junto con la asistencia a los seminarios formativos, suponen el cincuenta por ciento de la calificación del alumno en Pedagogía y el treinta por ciento en Educación Social.

Para la evaluación también se tiene en cuenta la calificación e informe que aporta el tutor profesional responsable de las prácticas en la institución correspondiente, en una nota ponderada que supone el cincuenta por ciento restante de la calificación en Pedagogía y el setenta por ciento en Educación Social.

\section{DiSEÑO DE LA INVESTIGACIÓN}

\subsection{Objetivos}

El estudio realizado tiene como objeto analizar la percepción hacia el Practicum del alumnado de último curso de Grado en Pedagogía y del Grado en Educación Social donde se ubican las prácticas curriculares, antes de empezar a realizar sus prácticas en una empresa, institución o centro educativo. Asimismo, se pretende conocer la valoración del proceso y gestión del Practicum, su propia percepción inicial sobre la utilidad de las prácticas externas, la valoración del alumnado 
SONIA CASILLAS MARTÍN, MARGARITA GONZÁLEZ SÁNCHEZ Y SARA SERRATE GONZÁLEZ PERCEPCIÓN DE LOS ESTUDIANTES DEL GRADO EN PEDAGOGÍA Y EDUCACIÓN SOCIAL SOBRE LA ORGANIZACIÓN, LA UTILIDAD Y FORMACIÓN DEL PRACTICUM

respecto a los criterios de evaluación de la asignatura Practicum y la formación recibida para afrontar estas prácticas a lo largo del Grado. A partir de los resultados obtenidos se persigue determinar si el proceso de organización y la metodología utilizada en la gestión del Practicum es adecuada desde el punto de vista del alumno o es susceptible de mejora. Se pretende, del mismo modo, poder disponer de evidencias sobre el proceso seguido, a partir de las cuales los coordinadores responsables académicamente del Practicum en las diferentes titulaciones puedan tomar decisiones con vistas a la mejora del proceso, puesta en marcha y criterios de calificación y formación del Practicum en los diversos grados examinados.

En este sentido, los objetivos específicos planteados en el estudio pretenden:

- Mejorar el Practicum de Educación Social y Pedagogía y analizar las posibilidades de enriquecer y mejorar el sistema de prácticas externas en Educación Superior.

- Analizar las diferencias en función de la titulación encontradas en las variables estudiadas.

- Identificar áreas de mejora en la organización de las prácticas externas.

- Conocer el grado de satisfacción del alumnado con la asignación del centro o institución en el que van a realizar las prácticas.

- Analizar su grado de conformidad con los aspectos organizativos de las prácticas.

- Conocer la percepción del alumnado sobre la utilidad de las prácticas en su formación profesional, antes de comenzarlas.

- Mejorar la evaluación del Practicum a partir de las opiniones de sus agentes principales, los alumnos.

- Conocer la valoración que tiene el alumnado acerca de la formación para el Practicum recibida a lo largo de la carrera.

\subsection{Metodología}

Dentro de los diferentes enfoques de investigación, nuestro trabajo se sitúa en el enfoque cuantitativo. Se ha elegido analizar los datos mediante una metodología de este carácter con el fin de concretar de forma más minuciosa algunos aspectos útiles para la mejora y calidad de la asignatura del Practicum, además de aportar datos valiosos para optimizar unas enriquecidas y valiosas prácticas externas. El diseño de investigación seleccionado, según los objetivos propuestos, es de tipo no experimental, y el método utilizado ha sido descriptivo-correlacional, a través del estudio de encuesta electrónica.

\subsection{Población y muestra}

La población de interés de nuestra investigación, tomando como criterio los objetivos planteados y de acuerdo con los datos facilitados por la Secretaría del 
SONIA CASILLAS MARTÍN, MARGARITA GONZÁLEZ SÁNCHEZ Y SARA SERRATE GONZÁLEZ PERCEPCIÓN DE LOS ESTUDIANTES DEL GRADO EN PEDAGOGÍA Y EDUCACIÓN SOCIAL SOBRE LA ORGANIZACIÓN, LA UTILIDAD Y FORMACIÓN DEL PRACTICUM

centro, ha quedado definida por los estudiantes que durante el curso 2014-2015 cursan los 24 créditos de la asignatura Practicum del Grado de Pedagogía y los 30 créditos del Grado de Educación Social de la Facultad de Educación de la Universidad de Salamanca. Está compuesta por 129 estudiantes, 62 son alumnado de Pedagogía y 67 de Educación Social. La muestra final quedó conformada por 101 estudiantes (56 de Pedagogía y 45 de Educación Social), que supone un 78\% de la población de referencia, lo que informa suficientemente de su representatividad, de los cuales, el 12,87\% son hombres y el $86,13 \%$ mujeres, con edades comprendidas entre los 21 y los 38 años. Todos ellos respondieron anónima y voluntariamente al cuestionario antes de comenzar las prácticas en el centro asignado para ello.

\subsection{Instrumento}

El instrumento de recogida de datos ha sido de naturaleza cuantitativa, en su mayor parte basado en la metodología de encuesta estructurada, dentro de la que incluimos, principalmente, escalas tipo Likert de 5 puntos, que permitiesen valorar cada ítem desde "totalmente en desacuerdo" hasta "totalmente de acuerdo». El cuestionario diseñado ad hoc, por el equipo de coordinación del Practicum, denominado "Percepción de los estudiantes de Pedagogía y Educación Social sobre la asignatura practicum» se aplicó a través de Internet (Díaz, 2012), en concreto desde el servicio de Google Drive.

El contenido del cuestionario, tal y como se recoge en la Tabla 1, consta de 25 ítems incluidos en cuatro dimensiones: a) datos de identificación, b) valoración del proceso y gestión del Practicum, c) percepción sobre la utilidad de las prácticas y d) criterios de evaluación del Practicum y formación recibida a lo largo del Grado.

TABLA 1

Contenido del cuestionario

\begin{tabular}{|c|c|}
\hline DIMENSIONES DEL CUESTIONARIO & NÚMERO DE ÍTEMS \\
\hline Datos de identificación & 4 \\
\hline Valoración del proceso y gestión del Practicum & 12 \\
\hline Utilidad de las prácticas & 4 \\
\hline $\begin{array}{c}\text { Criterios de evaluación del Practicum y } \\
\text { formación recibida a lo largo del Grado }\end{array}$ & \multicolumn{2}{|c|}{} \\
\hline
\end{tabular}

En cuanto a la validez de contenido, esta ha sido garantizada a través de la valoración por jueces expertos, profesorado universitario especialista tanto en metodología como en el tema de investigación trabajado y, algunos de ellos, con amplia y reconocida experiencia en la coordinación, organización y gestión de la asignatura del Practicum. Respecto a la consistencia interna del instrumento, la 
SONIA CASILLAS MARTÍN, MARGARITA GONZÁLEZ SÁNCHEZ Y SARA SERRATE GONZÁLEZ PERCEPCIÓN DE LOS ESTUDIANTES DEL GRADO EN PEDAGOGÍA Y EDUCACIÓN SOCIAL SOBRE LA ORGANIZACIÓN, LA UTILIDAD Y FORMACIÓN DEL PRACTICUM

correlación interelementos promedio arroja un coeficiente Alfa de Cronbach de 0,925 , lo que supone una fiabilidad muy adecuada para este tipo de instrumentos.

\subsection{Análisis de los datos}

Tras la cumplimentación del cuestionario por los estudiantes participantes, se ha llevado a cabo el análisis de datos mediante el paquete estadístico sPss v.21 (licencia Universidad de Salamanca). El tratamiento de los datos se ha centrado en un análisis descriptivo y comparativo de los resultados obtenidos de frecuencias, porcentajes y correlaciones. Estas últimas a través del coeficiente de Pearson, como método que mide la relación lineal entre las variables distribuidas normalmente. Una vez comprobados los supuestos paramétricos de normalidad (prueba de Kolmogorov-Smirnov), obteniendo en la misma una significación de 0,01 y siendo la distribución de la muestra normal, se optó por la utilización de pruebas paramétricas de contraste de hipótesis, en concreto el análisis de varianza factorial ANOvA, para comprobar las diferencias existentes entre los alumnos de pedagogía y de educación social respecto a si el proceso de elección y distribución de centros ha sido un proceso bien organizado.

\section{RESUlTAdos}

La exposición de los resultados obtenidos se ha organizado en torno a las dimensiones contempladas en el cuestionario como son la valoración del proceso y gestión del Practicum, la utilidad, la evaluación y formación, con la pretensión de ofrecer claridad y orden en la presentación de los mismos.

\subsection{Valoración del proceso y gestión del Practicum}

\subsubsection{Motivos de la elección de centro en prácticas}

Como se ha señalado, la coordinación del Practicum, en los meses previos al inicio de las prácticas externas, da la posibilidad al alumnado de elegir otro centro o institución que no se halle en el listado ofertado desde la Facultad. Por otro lado, realiza una oferta de centros y plazas donde aquellos estudiantes que, de forma autónoma, no han realizado su gestión de las prácticas, pueden realizarlas. En base a la relación de centros disponibles, los estudiantes seleccionan aquellas instituciones donde les gustaría realizar las prácticas de su titulación por orden de preferencia. 
SONIA CASILLAS MARTÍN, MARGARITA GONZÁLEZ SÁNCHEZ Y SARA SERRATE GONZÁLEZ PERCEPCIÓN DE LOS ESTUDIANTES DEL GRADO EN PEDAGOGÍA Y EDUCACIÓN SOCIAL SOBRE LA ORGANIZACIÓN, LA UTILIDAD Y FORMACIÓN DEL PRACTICUM

TABLA 2

Motivos de elección del centro en prácticas

\begin{tabular}{|c|c|c|}
\hline Motivos DE ELECCión DE CENTRO & \multicolumn{2}{|c|}{$\begin{array}{c}\text { Sí } \\
\text { (N-101) }\end{array}$} \\
\cline { 2 - 3 } Por especialidad que estoy cursando & $\mathrm{F}$ & $\%$ \\
Por temática & 13 & 12,9 \\
Por cercanía & 59 & 58,4 \\
\hline Porque conozco al responsable o algún trabajador & 22 & 21,8 \\
Porque creo que voy a aprender mucho sobre mi profesión & 11 & 10,9 \\
Otro 1: Dentro de las opciones era la que & 42 & 41,6 \\
más se ajustaba a mis preferencias & 4 & 4,0 \\
Otro 2: Porque me hablaron bien del centro & 3 & 3,0 \\
Otro 3: Porque estudié en ese centro & 1 & 1,0
\end{tabular}

En su mayoría, los motivos que llevan a elegir el centro en prácticas, tanto a los estudiantes de Pedagogía, como de Educación Social, son: la temática o colectivo con el que se trabaja (atención a personas con necesidades educativas, orientación, drogadicción, discapacidad, etc.) y el aprendizaje que piensan que les reportará en relación a aspectos básicos de su futura profesión. No obstante, hay que tener en cuenta que también se basan en criterios como la cercanía a la Facultad o a su lugar de residencia $(21,8 \%)$.

3.1.2. Proceso de asignación de centro en prácticas

TABLA 3

Resultados del proceso de asignación del centro en prácticas

\begin{tabular}{|l|c|c|c|c|}
\hline \multirow{2}{*}{ Proceso de ASIGNACIÓN DE CENTROS } & \multicolumn{2}{|c|}{ GRADO EN PEDAGOGÍA } & \multicolumn{2}{c|}{ GRADO EN EDUCACIÓN SOCIAL } \\
\cline { 2 - 5 } & $\mathrm{F}$ & $\%$ & $\mathrm{~F}$ & $\%$ \\
\hline De primera a tercera opción & 30 & 53,6 & 18 & 40,0 \\
Segunda vuelta & 7 & 12,5 & 1 & 2,2 \\
Asignada por coordinación & 5 & 8,9 & 1 & 2,2 \\
Elegido antes del proceso & 14 & 25,0 & 25 & 55,6 \\
Total & 56 & 100,0 & 45 & 100,0
\end{tabular}

Una vez que el alumnado establece sus preferencias, desde la coordinación del Practicum de ambas titulaciones se procede a asignar una plaza a cada alumno. Nos encontramos, en primer lugar, con que un $25 \%$ de los estudiantes 
SONIA CASILLAS MARTÍN, MARGARITA GONZÁLEZ SÁNCHEZ Y SARA SERRATE GONZÁLEZ PERCEPCIÓN DE LOS ESTUDIANTES DEL GRADO EN PEDAGOGÍA Y EDUCACIÓN SOCIAL SOBRE LA ORGANIZACIÓN, LA UTILIDAD Y FORMACIÓN DEL PRACTICUM

de Pedagogía y un 55,6\% de Educación Social eligen un centro no ofertado por la Facultad, antes del proceso de asignación. Excluyendo posteriormente a estos estudiantes, a los que no hay que asignar centro en prácticas, el 77,41\% de nuestro alumnado realizará sus prácticas en un centro que se encontraba entre la primera y la tercera opción de sus preferencias. A pesar de ser dos procesos distintos, los resultados muestran que, en ambas titulaciones, al alumnado se le ha asignado una plaza en algún centro que se encontraba entre sus prioridades. Es por ello que entendemos que trabajamos con una muestra con clara motivación hacia las prácticas que van a comenzar, lo que facilita encontrar una actitud positiva hacia las opiniones que se les pide.

\subsubsection{Satisfacción con el proceso}

En relación con la valoración que el alumnado tiene del proceso seguido en la asignación del centro de prácticas, las puntuaciones medias son elevadas, e indican que el 86,1\% de los estudiantes están satisfechos con el centro donde realizarán las prácticas y el $85,1 \%$ creen que el centro les va a permitir aprender más sobre la titulación que están cursando. Al menos el 38,7\% considera que el proceso de elección ha sido un proceso bien organizado, teniendo en cuenta que el 38,6\% responde que no se encuentran de acuerdo ni en desacuerdo con tal afirmación.

GRÁFICA 1

Proceso y grado de satisfacción con la asignación del centro en prácticas



En cuanto a la satisfacción y valoración general a estas tres afirmaciones, comprobamos que las puntuaciones son más altas en el Grado de Educación Social que en el de Pedagogía. Se observa, a partir de la Gráfica 1, que los estudiantes de 
SONIA CASILLAS MARTÍN, MARGARITA GONZÁLEZ SÁNCHEZ Y SARA SERRATE GONZÁLEZ PERCEPCIÓN DE LOS ESTUDIANTES DEL GRADO EN PEDAGOGÍA Y EDUCACIÓN SOCIAL SOBRE LA ORGANIZACIÓN, LA UTILIDAD Y FORMACIÓN DEL PRACTICUM

Pedagogía se manifiestan más en desacuerdo respecto a la afirmación el proceso de elección y distribución de centros ha sido bien organizado, encontrando diferencias estadísticamente significativas mediante la comparación de medias ( $\mathrm{F}=5,901$; Sig.= 017) que permiten afirmar que el alumnado del Grado en Educación Social está más de acuerdo que el alumnado del Grado en Pedagogía respecto a la afirmación, por ello puede considerarse que el alumnado prefiere el sistema del sorteo público antes que la asignación a través de la nota media.

Se ha encontrado, por otro lado, una correlación positiva $(\mathrm{r}=0,301 ; \mathrm{p}=0,002)$ respecto a las variables el proceso de elección y distribución de centro ba sido bien organizado y estoy satisfecho con el centro donde realizaré las prácticas, en este sentido afirmamos que el alumnado que considera que el proceso ha estado bien organizado son los estudiantes que están más satisfechos con el centro de prácticas.

\subsection{Utilidad de las prácticas}

Respecto a los aspectos por los que el alumnado considera que las prácticas resultarán más útiles, destaca el poder aplicar actitudes hacia la profesión en una situación profesional particular, aprender y aplicar habilidades interpersonales propias de la profesión, desarrollar competencias y capacidades importantes para su profesión y conocer mejor el ámbito profesional de su titulación. Por su parte, los estudiantes de Educación Social también consideran que las prácticas externas les van a permitir solucionar problemas prácticos habituales en el desarrollo de la profesión.

En general, el alumnado participante en el estudio considera que la realización de las prácticas externas contribuirá a su formación como profesional $(4,26)$, siendo destacable que la valoración es mayor entre los estudiantes de Educación Social que entre los de Pedagogía. 
SONIA CASILLAS MARTÍN, MARGARITA GONZÁLEZ SÁNCHEZ Y SARA SERRATE GONZÁLEZ

PERCEPCIÓN DE LOS ESTUDIANTES DEL GRADO EN PEDAGOGÍA Y EDUCACIÓN SOCIAL SOBRE LA ORGANIZACIÓN, LA UTILIDAD Y FORMACIÓN DEL PRACTICUM

\section{GRÁFICA 2}

Utilidad de las prácticas en el Grado de Pedagogía y Educación Social

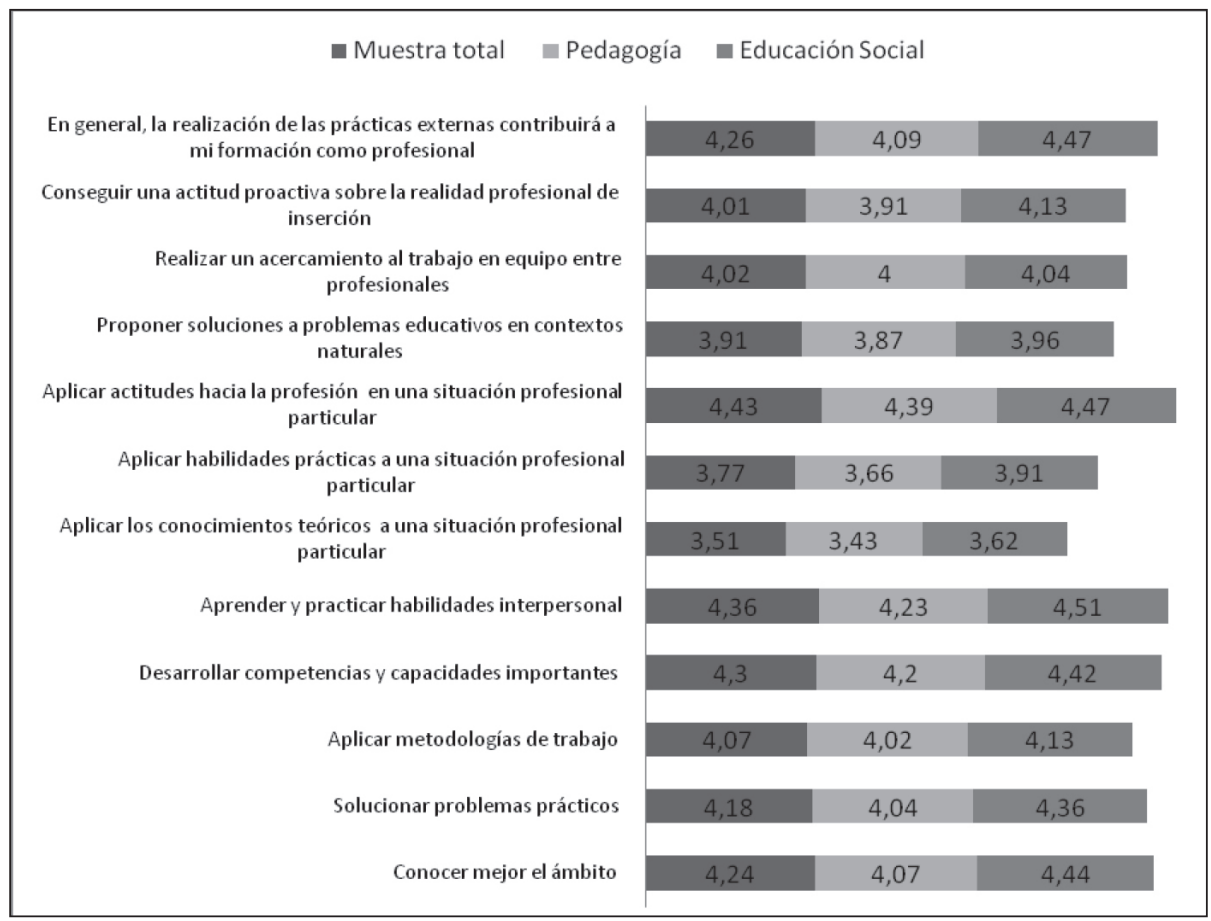

A partir de un análisis exploratorio se halla una correlación positiva $(r=0,766$; $\mathrm{p}=0,000$ ) en el sentido de que los estudiantes que consideran que el centro asignado les va a permitir aprender sobre la titulación que están cursando son los que creen que, en general, la realización de las prácticas externas contribuirá a su formación como profesional. En este sentido, se determina que tanto el centro asignado como la realización de las prácticas en sí mismas aportan al alumno una motivación y predisposición para seguir formándose y preparándose durante el tiempo que duran las prácticas, desde un marco distinto al académico, como es la realidad profesional. Considerar que las prácticas externas les van a ofrecer conocimientos, técnicas y habilidades profesionales supone un estímulo para el alumno que, no obstante, sería conveniente comprobar si se mantiene tras finalizar el periodo de prácticas externas. 
SONIA CASILLAS MARTÍN, MARGARITA GONZÁLEZ SÁNCHEZ Y SARA SERRATE GONZÁLEZ PERCEPCIÓN DE LOS ESTUDIANTES DEL GRADO EN PEDAGOGÍA Y EDUCACIÓN SOCIAL SOBRE LA ORGANIZACIÓN, LA UTILIDAD Y FORMACIÓN DEL PRACTICUM

3.3. Criterios de evaluación del Practicum y formación recibida a lo largo del Grado

GRÁFICA 3

Criterios de evaluación del Practicum y formación recibida a lo largo del Grado

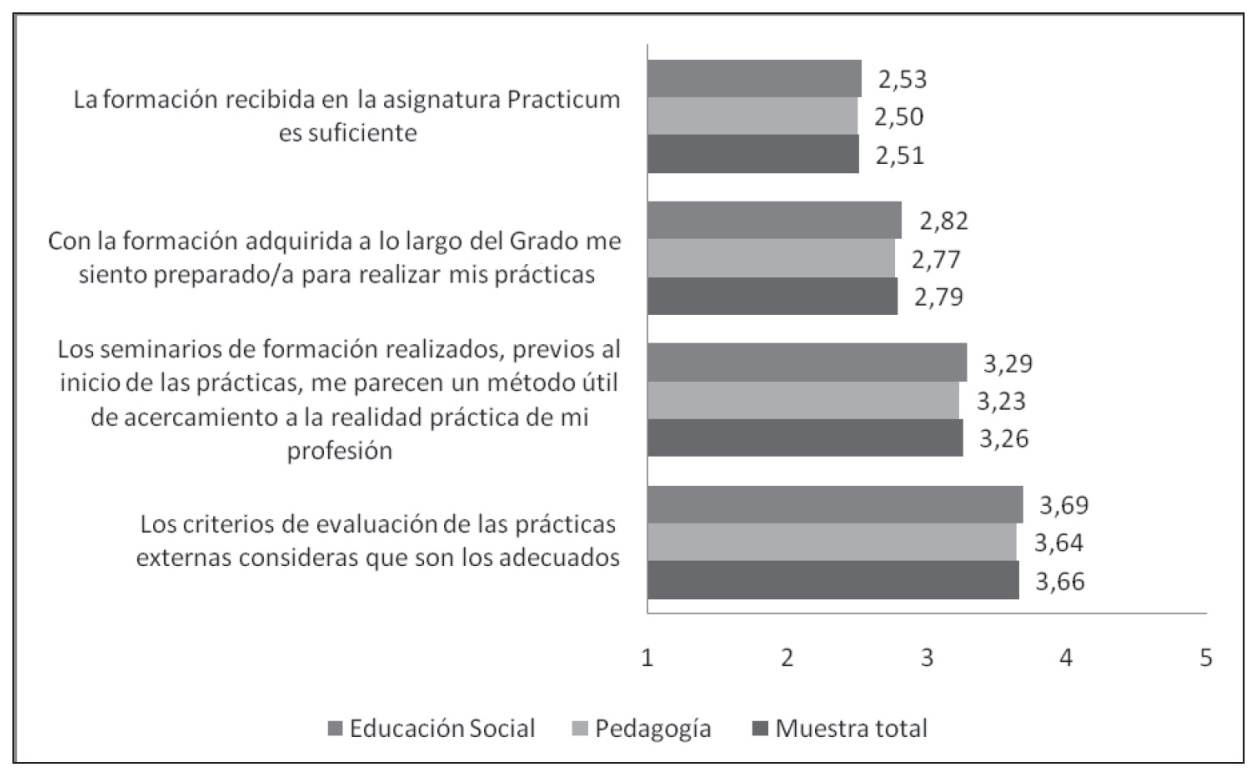

Podemos afirmar, a partir de los datos obtenidos, tal como se muestra en la Gráfica 3, que los estudiantes consideran que los criterios de evaluación de la asignatura Practicum son adecuados. Respecto a la formación para el Practicum, creen que los seminarios de formación, lecturas y reflexiones sobre las mismas son un método útil de acercamiento a la realidad profesional, no obstante, si nos centramos en la formación recibida a lo largo del Grado, el alumnado no se siente preparado para realizar las prácticas, al igual que no consideran que la formación específica para hacer frente a las prácticas de la titulación en la asignatura Practicum sea suficiente.

Del análisis exploratorio mencionado con anterioridad, se halla de nuevo una correlación positiva $(\mathrm{r}=0,376 ; \mathrm{p}=0,000)$ entre los estudiantes que creen poder aplicar conocimientos teóricos a una situación profesional particular, como criterio de utilidad, son aquellos que consideran que la formación recibida a lo largo del Grado les prepara para hacer frente a las prácticas de la titulación. Igualmente, estos estudiantes que creen poder aplicar mejor los conocimientos teóricos son nuevamente los que consideran que la formación recibida en la asignatura Practicum es suficiente $(\mathrm{r}=0,422 ; \mathrm{p}=0,000)$. 
SONIA CASILLAS MARTÍN, MARGARITA GONZÁLEZ SÁNCHEZ Y SARA SERRATE GONZÁLEZ PERCEPCIÓN DE LOS ESTUDIANTES DEL GRADO EN PEDAGOGÍA Y EDUCACIÓN SOCIAL SOBRE LA ORGANIZACIÓN, LA UTILIDAD Y FORMACIÓN DEL PRACTICUM

Es por ello que podemos afirmar que aunque la formación, tanto a lo largo del Grado, como la específica de la asignatura Practicum, es insuficiente desde la perspectiva del alumnado, aquellos que mejor preparados a nivel teórico se sienten son los que creen que la formación es suficiente y les prepara para hacer frente a las prácticas de la titulación. Sería conveniente, no obstante, conocer qué aspectos teóricos de los ya adquiridos durante la carrera creen que van a aplicar durante las prácticas, al igual que averiguar por qué determinados estudiantes consideran que la formación es insuficiente, en torno a los conocimientos teóricos y la preparación práctica que hubieran deseado adquirir durante el Grado y la asignatura Practicum.

\section{DisCUSIÓN DE RESULTADOS, CONCLUSIONES Y PROPUESTAS}

El estudio desarrollado nos permite reflexionar sobre determinadas cuestiones en relación al funcionamiento y desarrollo de la asignatura Practicum en las titulaciones de Pedagogía y Educación Social, y exponer algunas propuestas de actuación en determinadas áreas que permiten mejorar el planteamiento de dicha asignatura tan relevante en el currículum formativo de ambos grados.

A partir del análisis realizado queda patente que los estudiantes tienen un elevado grado de satisfacción, aunque mejorable en algunos aspectos, con el proceso y la distribución de centro en la asignatura Practicum. Se muestran muy satisfechos con el centro asignado para realizar sus prácticas externas, niveles que decrecen cuando se trata del proceso de elección de dicho centro. Se han encontrado diferencias significativas entre las percepciones que poseen los estudiantes de las dos titulaciones, siendo más altas las valoraciones del alumnado de Educación Social, en el que el proceso de asignación se desarrolla mediante sorteo público, frente al de Pedagogía que se establece por la nota media de los mismos en la titulación. Tal situación nos lleva a pensar que dichos estudiantes prefieren que sea la suerte la que designe su centro de prácticas, deduciendo que el proceso es más satisfactorio cuando se realiza por sorteo que por nota media, aunque es importante señalar que, la mayoría, cuando es por nota media, tienen el centro que han elegido entre sus primeras opciones.

La mayoría de los matriculados en el Practicum realizarán sus prácticas en uno de los centros o instituciones que se encontraba entre las tres primeras opciones de sus preferencias. Es por ello que consideramos trabajar con un alumnado que se encuentra muy motivado con la realización de las prácticas y, por lo tanto, con buena predisposición al aprendizaje. Este indicador será el pilar básico para que la satisfacción sea positiva en el desarrollo de las prácticas y sus creencias previas tengan que ver con unas altas expectativas.

Se ha comprobado que los estudiantes que se encuentran más de acuerdo con el hecho de que el proceso seguido en la distribución de centros ha estado bien organizado son los que afirman sentirse más satisfechos con el centro asignado para realizar las prácticas. Parece evidenciarse entonces que los que realizarán las prácticas en uno de los centros de sus preferencias tienden a valorar mejor 

PERCEPCIÓN DE LOS ESTUDIANTES DEL GRADO EN PEDAGOGÍA Y EDUCACIÓN SOCIAL SOBRE LA ORGANIZACIÓN, LA UTILIDAD Y FORMACIÓN DEL PRACTICUM

la organización de dichas prácticas. Algunos estudios como el de Morales (2013) afirman que los estudiantes muestran su insatisfacción hacia la organización del proceso por otros motivos relacionados con la falta de oferta formativa; en este sentido, hay que tener en cuenta que son muchas las variables que influyen en la valoración de este proceso, en determinadas ocasiones, a pesar de que una valoración sea negativa por parte del alumnado, no significa que sea indicador de una mala organización, sino de la influencia de multitud de variables ajenas a la organización tales como la nota obtenida en la asignatura, entre otras.

En nuestro estudio, tanto los estudiantes de Pedagogía como los de Educación Social, cuando se trata de valorar la satisfacción con el proceso seguido para asignar centro en prácticas, lo hacen de forma muy positiva. Al analizar los datos extraídos de las correlaciones en el conjunto de variables que miden la satisfacción con el proceso y asignación, encontramos que los estudiantes que consideran que, a priori, les va a permitir aprender sobre la titulación que está cursando creen que, en general, la realización de las prácticas externas contribuirá en mayor medida a su formación como profesional. Las creencias previas que el alumnado manifiesta antes de su incorporación a las prácticas son, por lo tanto, en su mayoría positivas. Esta motivación puede tener relación con el hecho de que realizarán las prácticas en uno de los centros que ellos habían seleccionado entre sus preferencias. En este sentido, el estudio realizado por Molina et al. (2004a), con los estudiantes de Magisterio, llega a conclusiones similares sobre las creencias previas que los estudiantes llevan consigo al Practicum.

Es preciso destacar, en cuanto a la importancia formativa de las prácticas externas, otras investigaciones en esta línea, como la de Zabalza (2004), donde se resalta que este momento forma parte del periodo de formación de los estudiantes. Es por ello que debe planificarse buscando la utilidad (González y Hevia, 2011) para el alumnado, especialmente para que el aprendizaje adquirido en este periodo sea significativo y duradero, ofreciendo oportunidades de formación profesional al alumnado en prácticas. Este sería el propósito principal de las prácticas y no otro, por este motivo es importante tener claro, a la hora de seleccionar y establecer los centros, instituciones y programas destinados a que el alumnado realice sus prácticas de titulación, qué tipo de formación se desea ofrecer y qué tipo de aprendizajes esperamos que el alumno adquiera.

Respecto a la formación recibida tanto a lo largo del Grado como la formación específica en la asignatura Practicum, los estudiantes piensan que es insuficiente y no les prepara para hacer frente a las prácticas externas. No obstante, los aspectos que valoran como más útiles de las prácticas son el poder «aplicar actitudes hacia la profesión" y "aprender y aplicar habilidades interpersonales", similares resultados que los señalados en el estudio de González y Laorden (2012). Se intuye, por lo tanto, que entre la formación recibida no son tanto los aspectos teóricos los que el alumnado cree que son insuficientes, como los aspectos prácticos y que fomenten el desarrollo de habilidades esenciales para formarse como profesionales. 
SONIA CASILLAS MARTÍN, MARGARITA GONZÁLEZ SÁNCHEZ Y SARA SERRATE GONZÁLEZ PERCEPCIÓN DE LOS ESTUDIANTES DEL GRADO EN PEDAGOGÍA Y EDUCACIÓN SOCIAL SOBRE LA ORGANIZACIÓN, LA UTILIDAD Y FORMACIÓN DEL PRACTICUM

Apoyan esta idea los resultados obtenidos en torno a que aquellos estudiantes que creen que con la formación obtenida a lo largo del Grado se sienten preparados para hacer frente a las prácticas de la titulación son los que consideran que van a poder aplicar mejor los conocimientos teóricos a una situación profesional particular. Esta valoración podría estar condicionada por el grado de implicación y rendimiento académico del alumno en esta titulación, considerando por tanto que los contenidos formativos prácticos en torno a habilidades y actitudes profesionales son los que el alumnado cree que no se desarrollan al mismo nivel que los conocimientos teóricos. En este sentido, hay estudios como el de González y Hevia (2010) que establecen una relación entre el binomio calificación-percepción, los estudiantes que obtienen mejores calificaciones son los que mejor percepción tienen de las prácticas e incluso de su formación ante estas y, por lo tanto, de su capacidad para afrontar este periodo.

Este hecho podría explicar que el alumnado que en nuestro estudio cree poder aplicar mejor los conocimientos teóricos a una situación real concreta y específica es aquel que considera que la formación recibida para hacer frente a las prácticas de la titulación es suficiente, sintiéndose formado para actuar como profesionales para enfrentarse a situaciones que requieren una intervención socioeducativa. Por tanto, concluir, de acuerdo con Molina et al. (2004b), que la relación entre teoríapráctica debe estar vinculada, en tanto el sentimiento que manifiesta el alumnado y la percepción de su propia capacidad como profesional va a depender no solo de conocimientos teóricos, sino de la preparación y puesta en práctica de esos conocimientos, desde las facultades y, en la medida de lo posible, desde las aulas, de forma que el alumno pueda sentirse más seguro a la hora de hacer frente a las prácticas externas de la titulación.

En este sentido, Pérez et al. (2006: 37) proponen que el Practicum se consolide en tres aspectos cruciales: por un lado, como estrategia de formación permanente para los tutores profesionales; por otro lado, como herramienta para consolidar la investigación del alumno, y, por último, como instrumento para la mejora del desarrollo profesional del profesor universitario. Propone que los docentes universitarios deben profundizar en cómo pasar de los aprendizajes teóricos a los prácticos y su conexión; y todo ello con el fin de mostrar cómo el ejercicio de una supervisión reflexiva estimula la reflexión en los estudiantes tutorizados y en el propio supervisor durante el periodo de Practicum.

Es reseñable que, en relación a los criterios de evaluación planteados en la asignatura, el alumnado considera que son los adecuados y que, además, los seminarios de formación, lecturas y reflexiones empleados en la materia son un método útil de acercamiento a la realidad práctica. Aun así, y teniendo en cuenta que el alumnado considera insuficiente la formación en la asignatura Practicum, sería necesario establecer planes de formación más exhaustivos y especializados en torno al trabajo que desempeñarán los futuros pedagogos y educadores sociales en función de las diferentes salidas profesionales. 
SONIA CASILLAS MARTÍN, MARGARITA GONZÁLEZ SÁNCHEZ Y SARA SERRATE GONZÁLEZ PERCEPCIÓN DE LOS ESTUDIANTES DEL GRADO EN PEDAGOGÍA Y EDUCACIÓN SOCIAL SOBRE LA ORGANIZACIÓN, LA UTILIDAD Y FORMACIÓN DEL PRACTICUM

Entre las propuestas de mejora que se desprenden del estudio, cabe destacar las siguientes:

- Ampliar el estudio del nivel de satisfacción con la asignatura de Practicum a otros agentes implicados en la asignatura, como son los tutores profesionales, académicos y coordinadores.

- Utilizar instrumentos de carácter más cualitativo que permitan ampliar la información del cuestionario aplicado y, por lo tanto, medir, aún más, aspectos relacionados con la percepción real del alumnado en cuanto a la preparación, formación y satisfacción en los periodos programados al inicio de las prácticas.

- Aumentar el estudio en el tiempo, permitiendo que se puedan comparar resultados de diferentes promociones y de diferentes titulaciones de la Facultad.

- Realizar un postest en el que se recojan las percepciones del alumnado una vez finalizadas las prácticas, y poder comparar estas valoraciones con las recogidas antes del inicio del periodo de prácticas. Esta información nos permitirá ver si el grado de satisfacción con el proceso, con la asignación de centros y con el centro asignado se corresponde con la satisfacción tras haber finalizado el periodo de prácticas.

- Actualizar los materiales utilizados para la asignatura de Practicum, así como hacer guías de seguimiento para establecer relaciones entre los tutores profesionales y los tutores académicos y estandarizar los protocolos en todas las titulaciones de la Facultad de Educación.

- Implicar, en mayor medida, a los tutores profesionales y académicos en el proceso, para que ambas partes consigan hacer un fiel seguimiento de las prácticas de los estudiantes. Fijando obligatoriamente tutorías en los momentos claves de las prácticas (previas, inicio, seguimiento y final).

En esta línea, y con la misma finalidad que nuestro trabajo, otras investigaciones recientes, como la de Rial y Barreira (2012: 171), proponen mejorar la calidad del Practicum en relación a la profesionalización de sus estudiantes atendiendo a diversos aspectos. En primer lugar, señalan la delimitación de las funciones y perfiles propios, lo que marcará las exigencias al alumno durante su periodo de prácticas; por otro lado, el análisis, periódicamente, de las empresas o centros que colaboren para constatar la presencia de la figura de profesional preparado; un cambio en las condiciones de los docentes universitarios que supervisan las prácticas, tales como reducción de docencia en otras materias curriculares, provisión de facilidades de coordinación con el alumnado o contratación de más personal (de ayuda en la gestión o en la tarea docente). Asi mismo la ampliación del seguimiento y colaboración desde la Universidad, mediante contactos más continuados, con el centro y con el alumnado. Actualmente las TIC pueden facilitar esta tarea. Además de una ampliación temporal del periodo de prácticas de manera que la experiencia laboral adquirida sea más abundante. Y, por último, la reestructuración 
SONIA CASILLAS MARTÍN, MARGARITA GONZÁLEZ SÁNCHEZ Y SARA SERRATE GONZÁLEZ PERCEPCIÓN DE LOS ESTUDIANTES DEL GRADO EN PEDAGOGÍA Y EDUCACIÓN SOCIAL SOBRE LA ORGANIZACIÓN, LA UTILIDAD Y FORMACIÓN DEL PRACTICUM

del Practicum, teniendo en cuenta la implicación de otros agentes en el diseño y estructuración curricular, como agentes sociales, administraciones e incluso colegios profesionales, agrupándolos en equipos de trabajo (comisión, cuerpo representativo...).

\section{REFERENCIAS BIBLIOGRÁFICAS}

Armengol, C. et al. (2011). El Practicum en el Espacio Europeo de Educación Superior (EEES): mapa de competencias del profesional de la educación. Revista de Educación, 354, $71-98$.

Bas, E. (2005). El Practicum en las titulaciones de Pedagogía y Educación Social: entre el discurso y sus prácticas. Education, 23, 191-206.

Darreche, L.; Ordeñana, M. B. y Beloki, N. (2011). La potencialidad del Practicum en el desarrollo profesional de los educadores y educadoras sociales: una llamada a su revalorización. RES. Revista de Educación Social, 13, 1-11. Recuperado el 29 de enero. http://www.eduso.net/res/.

Díaz, V. (2012). Ventajas e inconvenientes de la encuesta por Internet. Papers, 97/1, 193-223.

Gairín, J. y Martín, M. (2004). Las instituciones educativas en la encrucijada de los nuevos tiempos: retos, necesidades, principios y actuaciones. Tendencias Pedagógicas, 9, 21-44. Recuperado el 10 de febrero de http://www.tendenciaspedagogicas.com/Articulos/2004_09_01.pdf.

González, M. (2009). Integración de práctica y reflexión en un único proceso. Revista Portuguesa de Pedagogía, vol. 43 (2), 23-40.

http://dx.doi.org/10.14195/1647-8614_43-2_2

González, T. (2007). El concepto de «buenas prácticas»: origen y desarrollo. Revista Comunicación y Pedagogía, 222, 32-35.

González, X. A. y Hevia, I. (2011). El Practicum de la Licenciatura de Pedagogía: estudio empírico desde la perspectiva del alumnado. Revista de Educación, 354, enero-abril, 209-236. Recuperado el 29 de enero. http://www.mecd.gob.es/revista-de-educacion/.

González-Garzón, M. L. y Laorden, C. (2012). El Practicum en la formación inical de los Maestros en las nuevas titulaciones de Educación Infantil y Primaria. El punto de vista de profesores y alumnos. Pulso, 35, 131-154.

Latorre, M. J. (2007). El potencial formativo en las creencias que sobre la enseñanza práctica poseen los futuros maestros. Revista de Educación, 343, mayo-agosto, 249-273. Recuperada el 29 de enero. http://www.mecd.gob.es/revista-de-educacion/.

López, M. C. e Hinojosa, E. (2008). Percepciones iniciales de los estudiantes sobre la formación práctica. Revista Iberoamericana de Educación, 47/5, 1-12. Recuperado el 29 de enero. http://www.rieoei.org/index.php.

Martínez, M. E. (2007). El portafolio del estudiante: Herramienta de enseñanza-aprendizaje y evaluación en ECTs. En A. Barca y otros. IX Congreso Internacional Galego-Portugués de Psicopedagogía. A Coruña: Universidad de A Coruña.

Molina, E. et al. (2004a). Formación práctica de los estudiantes de pedagogía en las Universidades Españolas. Profesorado, Revista de Currículum y Formación del Profesorado, 8 (2), 1-24. Recuperado el 10 de febrero. http://www.ugr.es/ recfpro/rev82ART3.pdf. 

SOBRE LA ORGANIZACIÓN, LA UTILIDAD Y FORMACIÓN DEL PRACTICUM

Molina, E. et al. (2004b). La mejora del Practicum, esfuerzo de colaboración. Profesorado, Revista de Currículum y Formación del Profesorado, 8 (2), 1-31. Recuperado el 10 de febrero. http://www.ugr.es/ recfpro/rev82ART4.pdf.

Morales, S. (2010). El Practicum en educación social: el portafolio como instrumento de seguimiento y evaluación del aprendizaje de los alumnos. Revista Docencia e Investigación, 20, 33-58. Recuperado el 10 de febrero. http://www.uclm.es/varios/revistas/ docenciaeinvestigacion/pdf/numero10/2.pdf.

Morales, S. (2013). Evaluando el practicum en Educación Social: acciones de mejora ante la puesta en práctica de los nuevos grados. Revista de Docencia Universitaria. REDU, vol. 11 (1), enero-abril, 349-364. Recuperado el 29 enero. http://www.red-u.net/.

Pérez García, M. ${ }^{a}$ P.; Benarroch Benarroch, A.; Jiménez Jiménez, M. ${ }^{a}$ Á.; Smith Fernández, G. y Rojas Ruiz, G. (2006). ¿¿Se puede estimular la reflexión en el supervisor y en el alumno universitario durante el periodo de prácticum? Enseñanza \& Teaching, 24, 33-51.

Rial Sánchez, A. F. y Barreira Cerqueiras, E. M. (2012). El Prácticum de la titulación de Pedagogía desde el punto de vista profesional. Enseñanza E Teaching, 30 (2), 153-174.

Woolfolk, A. y Murphy, P. K. (2001). Teaching educational psycology to de implicit mind. En R. Sternberg y B. Torff (eds.). Understanding and teaching the Implicit Ming (pp. 145-185). Mahwah, N. J.: Erlbaum.

Zabalza, M. A. (2004). Condiciones para el desarrollo del Practicum. Profesorado, Revista de Currículum y Formación del Profesorado, 8 (2), 1-22. Recuperado el 10 de febrero. http://www.ugr.es/ recfpro/rev82ART1.pdf.

Zabalza, M. A. (2006). El Practicum y la formación del profesorado: balance y propuestas para las nuevas titulaciones. En J. M. Escudero (Coord.). La mejora de la educación y la formación del profesorado. Políticas y prácticas (pp. 309-330). Barcelona: Octaedro.

Zabalza, M. A. (2011). El Practicum en la formación universitaria: estado de la cuestión. Revista de Educación, 354, 21-43. Recuperado el 10 de febrero. http://www.revistaeducacion.mec.es/re354/re354_02.pdf. 